Onno R. Diermann*

\title{
Mathieu-state reordering in periodic thermodynamics
}

https://doi.org/10.1515/zna-2021-0253

Received August 29, 2021; accepted August 30, 2021;

published online September 17, 2021

Abstract: A periodically driven, moderately anharmonic oscillator constitutes an ideal model system for investigating quantum resonances, which are amenable to a quantum pendulum approximation. In the present paper, I study the quasi-stationary Floquet-state occupation probabilities which emerge when such a resonantly driven system is coupled to a heat bath. It is demonstrated that the Floquet state which is associated with the ground state of the pendulum turns into an effective ground state, carrying the highest population in the strong-driving regime. Moreover, the population of this effective Floquet ground state can even exceed that of the undriven system's true ground state at the same bath temperature. These effects can be optimized by suitably engineering the properties of the bath.

Keywords: Floquet states; Mathieu approximation; nonequilibrium steady state; periodically driven quantum systems; quantum resonance; quasistationary distribution.

\section{Introduction}

The subject of nonequilibrium steady states of periodically driven open quantum systems has been intensely discussed in the scientific community over the last years. Here, the recent theoretical description $[1,2]$ and experimental observation [3, 4] of Floquet time crystals comes to mind as a prominent example. In these systems, the spontaneous breaking of the discrete time translation symmetry is related to many-body localization, with the help of Floquet theory. In contrast, in the present work, I describe a peculiar quantum-statistical effect that occurs already in periodically driven single-particle systems in contact with a heat bath. Such open Floquet systems have been

\footnotetext{
*Corresponding author: Onno R. Diermann, Carl von Ossietzky Universität, Institut für Physik, D-26111, Oldenburg, Germany,

E-mail: onno@ewe.net
}

studied for quite some time [5-7]. The underlying theoretical framework has been concisely summarized by Kohn [8], and been dubbed "Periodic Thermodynamics". First and foremost, this theory allows one to determine a quasistationary distribution of the occupation probabilities of the driven system's Floquet states; such distributions can differ significantly from a canonical equilibrium distribution. This toolbox has been applied by several authors to a rich variety of problems [9-14]; in particular, it has been argued that, somewhat counterintuitively, the driven system may effectively be cooled [15-17].

In principle, a quantum system which is exposed to a strong time-periodic driving force with angular frequency $\omega$ does not possess a ground state in the usual sense. This is related to the fact that the quasienergies of the system's Floquet states form Brillouin zones of width $\hbar \omega$, so that the quasienergy spectrum is unbounded from below. The aim of the present paper is to demonstrate that a quantum resonance actually can lead to an ordering of a part of the Floquet states, implying that an effective Floquet ground state emerges which carries most of the population in the nonequilibrium steady state, and which is not connected to the ground state of the undriven system by continuity when the driving force is switched off. To achieve this goal, I resort to the single-particle model of a driven anharmonic oscillator which is coupled to a heat bath, and analyze the effect of the driving force on the quasi-stationary distribution of Floquet-state occupation probabilities. This model is as simple as possible, and as complicated as necessary, for studying the role of quantum resonances in Periodic Thermodynamics with a view on the quantum-classical correspondence [10, 18, 19].

The quantum mechanical driven anharmonic oscillator describes a particle of mass $m$ confined by an onedimensional potential with a quartic anharmonicity and subjected to a monochromatic driving force, as defined by the Hamiltonian

$$
H(t)=-\frac{\hbar^{2}}{2 m} \frac{\mathrm{d}^{2}}{\mathrm{~d} x^{2}}+\frac{1}{2} m \omega_{0}^{2} x^{2}+\frac{1}{4} g x^{4}+\Lambda x \cos (\omega t) .
$$

Here, the coefficient $g$ determines the strength of the anharmonicity, and $\omega_{0}$ is the oscillation frequency for $g=0$ and $\Lambda=0$, with $\Lambda$ denoting the forcing strength. Employing the harmonic-oscillator length $a=\sqrt{\hbar /\left(m \omega_{0}\right)}$ for introducing the dimensionless coordinate $q=x / a$, and 
invoking the dimensionless time $\tau=\omega t$, the Hamiltonian takes the convenient form

$$
H(\tau)=\hbar \omega_{0}\left(-\frac{1}{2} \frac{\mathrm{d}^{2}}{\mathrm{~d} q^{2}}+\frac{1}{2} q^{2}+\frac{\beta}{4} q^{4}+\lambda q \cos (\tau)\right)
$$

with dimensionless parameters $\beta=\hbar g /\left(m^{2} \omega_{0}^{3}\right)$ and $\lambda=$ $\Lambda / \sqrt{\hbar m \omega_{0}^{3}}$ now encoding the anharmonicity and the driving strength, respectively.

The quantum dynamics of this system are determined by its Floquet states $[20,21]$

$$
\left|\psi_{n}(q, \tau)\right\rangle=\left|u_{n}(q, \tau)\right\rangle \exp \left(-\mathrm{i} \frac{\varepsilon_{n}}{\hbar \omega} \tau\right),
$$

which are obtained by solving the eigenvalue equation

$$
\left(H(\tau)-\mathrm{i} \hbar \omega \frac{\partial}{\partial \tau}\right)\left|u_{n}(q, \tau)\right\rangle=\varepsilon_{n}\left|u_{n}(q, \tau)\right\rangle
$$

in an appropriate extended Hilbert space [22] for the periodic Floquet functions $\left|u_{n}(q, \tau)\right\rangle=\left|u_{n}(q, \tau+2 \pi)\right\rangle$ and their quasienergies $\varepsilon_{n}$. If $\varepsilon_{n}$ is an eigenvalue of the quasienergy operator $\left(H(\tau)-\mathrm{i} \hbar \omega \partial_{\tau}\right)$, so is $\varepsilon_{n}+\ell \hbar \omega$ for any positive or negative integer $\ell$, which means that the quasienergy pertaining to a Floquet state (3) labeled by $n$ is defined only modulo $\hbar \omega$. Thus, one representative of the quasienergies of each Floquet state falls into the interval $-1 / 2 \leq \varepsilon /(\hbar \omega)<+1 / 2$, and into any other Brillouin zone, which renders a rigorous mathematical solution of the quasienergy eigenvalue problem for the driven anharmonic oscillator (2) exceedingly difficult; it is expected that its quasienergies constitute a dense pure point spectrum [23-25].

The corresponding classical dynamics can be inferred from the scaled Hamiltonian system

$$
\begin{aligned}
& \frac{\mathrm{d} q}{\mathrm{~d} \tau}=P \\
& \frac{\mathrm{d} P}{\mathrm{~d} \tau}=-v^{-2}\left(q+\beta q^{3}+\lambda \cos (\tau)\right)
\end{aligned}
$$

with a dimensionless momentum variable $P$ which is related to the physical momentum $p$ through the relation $P=p /(m \omega a)$, and $v=\omega / \omega_{0}$ denoting the ratio of the driving frequency to the harmonic oscillation frequency. In the $(q, P)$-plane of the scaled phase space, a quantum state then occupies the area

$$
2 \pi \hbar_{\mathrm{eff}}=\frac{2 \pi \hbar}{(p / P)(x / q)}=\frac{2 \pi \hbar}{m \omega a^{2}}=2 \pi v^{-1},
$$

so that the "effective action quantum" $\hbar_{\text {eff }}=v^{-1}$ now replaces Planck's constant $\hbar$.

\section{The Mathieu approximation}

While the quasienergy eigenvalue problem (4) cannot be solved analytically for the driven anharmonic oscillator, there exists a universal resonance approximation which can be applied to periodically driven quantum systems of the general form (1), that is,

$$
H(t)=H_{0}+\Lambda x \cos (\omega t),
$$

provided that the energy eigenvalues $E_{n}$ of the undriven system $H_{0}$, obtained by solving the stationary Schrödinger equation $H_{0}|n\rangle=E_{n}|n\rangle$, vary sufficiently slowly with the quantum number $n$, so that the formal derivatives $E_{n}^{\prime} \equiv$ $\mathrm{d} E / \mathrm{d} n$ can be meaningfully taken. Moreover, it is assumed that there exists a "resonant" state $n=r$ which is singled out by the condition

$$
\hbar \omega \stackrel{!}{=} E_{r}^{\prime} \approx E_{r+1}-E_{r} .
$$

These requirements can be well satisfied by the driven anharmonic oscillator (1) for sufficiently large $v>1$, or correspondingly small $\hbar_{\text {eff }}$, placing the system into the semiclassical regime, implying $r \gg 1$. With respect to the corresponding classical system, the resonance condition (8) means that there is a 1:1 resonance, such that the time $T=2 \pi / \omega$ of one driving cycle is close to the time required for one oscillation of the undriven particle in the anharmonic potential. The following steps therefore can be understood as a quantum mechanical version of the familiar classical pendulum approximation [26]. They originate in a brief sketch by Berman and Zaslavsky [27], and have been worked out in similar form in Refs. [19, 28].

Under the conditions specified above, a natural ansatz for a Floquet state is given by [27]

$$
\left.\left|\psi(t)=\mathrm{e}^{-\mathrm{i} \eta t / \hbar} \sum_{n} b_{n}\right| n\right\rangle \exp \left[-\frac{\mathrm{i}}{\hbar}\left(E_{r}+(n-r) \hbar \omega\right) t\right],
$$

where the coefficients $b_{n}$ and the contribution $\eta$ to the quasienergy still need to be determined. Inserting this ansatz into the time-dependent Schrödinger equation gives

$$
\begin{aligned}
\mathrm{i} \hbar \frac{\partial}{\partial t}|\psi(t)\rangle= & \mathrm{e}^{-\mathrm{i} \eta t / \hbar} \sum_{n}\left(\eta+E_{r}+(n-r) \hbar \omega\right) b_{n}|n\rangle \\
& \times \exp \left[-\frac{\mathrm{i}}{\hbar}\left(E_{r}+(n-r) \hbar \omega\right) t\right]
\end{aligned}
$$

on the one hand, and

$$
\begin{aligned}
H(t)|\psi(t)\rangle= & \mathrm{e}^{-\mathrm{i} t / \hbar} \sum_{n}\left(E_{n}+\Lambda x \cos (\omega t)\right) b_{n}|n\rangle \\
& \times \exp \left[-\frac{\mathrm{i}}{\hbar}\left(E_{r}+(n-r) \hbar \omega\right) t\right]
\end{aligned}
$$


on the other, yielding the system of equations

$$
\begin{aligned}
\eta b_{m}= & \left(E_{m}-E_{r}-(m-r) \hbar \omega\right) b_{m} \\
& +\Lambda \cos (\omega t) \sum_{n}\langle m|x| n\rangle b_{n} \mathrm{e}^{\mathrm{i}(m-n) \omega t} .
\end{aligned}
$$

With the help of three approximations this system can be simplified to give an approximate analytical solution to the quasienergy eigenvalue problem: firstly, the energy eigenvalues of $H_{0}$ are expanded to second order,

$$
E_{m} \approx E_{r}+(m-r) \hbar \omega+\frac{E_{r}^{\prime \prime}}{2}(m-r)^{2},
$$

again relying on the requirement that the exact eigenvalues vary only slowly with the quantum number, and exploiting the resonance condition (8). Secondly, in a secular approximation akin to the rotating wave approximation, only the stationary terms $n=m \pm 1$ are kept in system (12), reducing the couplings among the $b_{m}$ to those between nearest neighbors only,

$$
\begin{aligned}
\eta b_{m}= & \frac{1}{2}(m-r)^{2} E_{r}^{\prime \prime} b_{m} \\
& +\frac{\Lambda}{2}\left(\langle m|x| m-1\rangle b_{m-1}+\langle m|x| m+1\rangle b_{m+1}\right) .
\end{aligned}
$$

Thirdly, all remaining dipole matrix elements are approximated by a constant,

$$
\langle m|x| m \pm 1\rangle \approx\langle r|x| r-1\rangle,
$$

which is viable for $r \gg 1$. As a result, one is left with the system

$$
\begin{aligned}
\eta b_{m}= & \frac{1}{2}(m-r)^{2} E_{r}^{\prime \prime} b_{m} \\
& +\frac{\Lambda}{2}\langle r|x| r-1\rangle\left(b_{m-1}+b_{m+1}\right) .
\end{aligned}
$$

Representing the desired coefficients $b_{m}$ as the Fourier coefficients of a $2 \pi$-periodic function $f(\vartheta)$, such that the index $m=r$ corresponds to the zero mode, one has

$$
b_{m}=\frac{1}{2 \pi} \int_{0}^{2 \pi} \mathrm{d} \vartheta f(\vartheta) \mathrm{e}^{-\mathrm{i}(m-r) \vartheta},
$$

implying the identity

$$
\begin{aligned}
(m-r)^{2} b_{m} & =-\frac{1}{2 \pi} \int_{0}^{2 \pi} \mathrm{d} \vartheta f(\vartheta) \frac{\mathrm{d}^{2}}{\mathrm{~d} \vartheta^{2}} \mathrm{e}^{-\mathrm{i}(m-r) \vartheta} \\
& =-\frac{1}{2 \pi} \int_{0}^{2 \pi} \mathrm{d} \vartheta f^{\prime \prime}(\vartheta) \mathrm{e}^{-\mathrm{i}(m-r) \vartheta}
\end{aligned}
$$

by the virtue of a twofold integration by parts, exploiting the $2 \pi$-periodicity of $f(\vartheta)$. Thus, the approximate nearestneighbor system (16) is transformed into the second-order differential equation

$$
\eta f(\vartheta)=-\frac{1}{2} E_{r}^{\prime \prime} f^{\prime \prime}(\vartheta)+\Lambda\langle r|x| r-1\rangle \cos (\vartheta) f(\vartheta)
$$

which may be interpreted as the stationary Schrödinger equation for a fictitious particle moving in a cosine well with periodic boundary conditions, i.e., a pendulum. The depth of the cosine well is determined by the driving strength $\Lambda$, while the mass of the fictitious particle is proportional to $1 / E_{r}^{\prime \prime}$ : the smaller the anharmonicity, the larger the mass.

The further minor transformation $\vartheta=2 z$ and $f(2 z)$ $\equiv \chi(z)$ produces

$$
\left(\frac{\mathrm{d}^{2}}{\mathrm{~d} z^{2}}+\frac{8 \eta}{E_{r}^{\prime \prime}}-2 \frac{4 \Lambda}{E_{r}^{\prime \prime}}\langle r|x| r-1\rangle \cos (2 z)\right) \chi(z)=0,
$$

which is the standard form of the famous Mathieu equation [29]

$$
\left(\frac{\mathrm{d}^{2}}{\mathrm{~d} z^{2}}+\alpha-2 Q \cos (2 z)\right) \chi(z)=0
$$

with parameters

$$
\alpha=\frac{8 \eta}{E_{r}^{\prime \prime}}
$$

and

$$
Q=\frac{4 \Lambda}{E_{r}^{\prime \prime}}\langle r|x| r-1\rangle .
$$

As is well known, the required $\pi$-periodic Mathieu functions $\chi(z)=\chi(z+\pi)$ exist only for certain characteristic values of $\alpha$; these values are usually designated [29] as $a_{0}, a_{2}, a_{4}, \ldots$ and $b_{2}, b_{4}, \ldots$ for even and odd functions, respectively. Thus, introducing a new quantum number $k=0,1,2,3, \ldots$, and writing

$$
\alpha_{k}(Q)= \begin{cases}a_{k}(Q), & k=0,2,4, \ldots \\ b_{k+1}(Q), & k=1,3,5, \ldots,\end{cases}
$$

the energy eigenvalues of the pendulum (19) for a given driving strength $\Lambda$, that is, for a given value of the Mathieu parameter (23), take the form

$$
\eta_{k}=\frac{1}{8} E_{r}^{\prime \prime} \alpha_{k}(Q) .
$$

Reverting these steps, and resubstitution into the ansatz (9), finally delivers the searched-for Mathieu approximation for the near-resonant Floquet states,

$$
\left|\psi_{k}^{(r)}(t)\right\rangle=\exp \left(-\frac{\mathrm{i}}{\hbar}\left(E_{r}+\eta_{k}\right) t\right) \sum_{\ell} f_{\ell, k}|r+\ell\rangle \mathrm{e}^{-\mathrm{i} \ell \omega t},
$$


where $f_{\ell, k}$ denotes the $\ell$ th Fourier coefficient of the associated $k$ th Mathieu function; according to Eq. (25), their quasienergies are given by

$$
\varepsilon_{k}^{(r)}=E_{r}+\frac{1}{8} E_{r}^{\prime \prime} \alpha_{k}(Q) \quad \bmod \hbar \omega .
$$

These states are labeled here by the quantum number $r$ of the anharmonic-oscillator state which is resonant due to the choice (8) of the driving frequency, and by the quantum number $k$ of the energy eigenstates of the pendulum (19). It is this quantum number $k$ which introduces a natural order of the near-resonant Floquet states. I will demonstrate in Sections 4 and 5 that this ordering actually is highly significant for Periodic Thermodynamics. Before, the reliability of the above general approximation scheme will be ascertained for the particular model system (2) of the driven anharmonic oscillator.

To do so, the Floquet states and their quasienergies are computed numerically by diagonalizing the one-cycle evolution operator within a basis of $b$ harmonic-oscillator eigenstates. In Figure 1 the quasienergy spectra obtained in this manner for $\beta=0.1$ and varying $\lambda$ (black lines) are compared with their Mathieu approximations (27) (dashed red lines). The upper panel refers to $r=10(v=1.4508$; basis size $b=41)$, the lower panel to $r=20(v=1.6909 ; b=61)$. In both cases the approximation is remarkably good for low Mathieu quantum numbers $k$ and gradually deteriorates with increasing $k$; moreover, the approximation is even better for $r=20$ than for $r=10$, as expected.

The fact that the "resonant" Floquet state with Mathieu quantum number $k=0$ actually does acquire features of an effective ground state is illustrated further by Figure 2, which shows contour plots of the probability density of the approximate Floquet states (26) with $k=0$ for $\beta=0.9$ and $r=10(v=2.577)$, for both $\lambda=0.01$ (upper panel) and $\lambda=1$ (lower panel). Under weak driving, $\lambda=0.01$, the time evolution of the probability density evidently is that of the anharmonic-oscillator eigenstate with $n=r=10$ which responds merely perturbatively to the driving force. Under strong driving, however, $\lambda=1$, the probability density is that of a single-humped wave packet which follows the stable classical periodic orbit brought about by the 1:1resonance according to the Poincaré-Birkhoff-theorem, reflecting the ground state of the pendulum (19) set in motion. Thus, the metamorphosis undergone by this Floquet state with increasing driving strength is another indication of the reordering of states effectuated by a quantum resonance, with the resonant eigenstate of the anharmonic oscillator turning into an effective Floquet ground state.

The interpretation of this resonant Floquet state with Mathieu quantum number $k=0$ as an effective ground
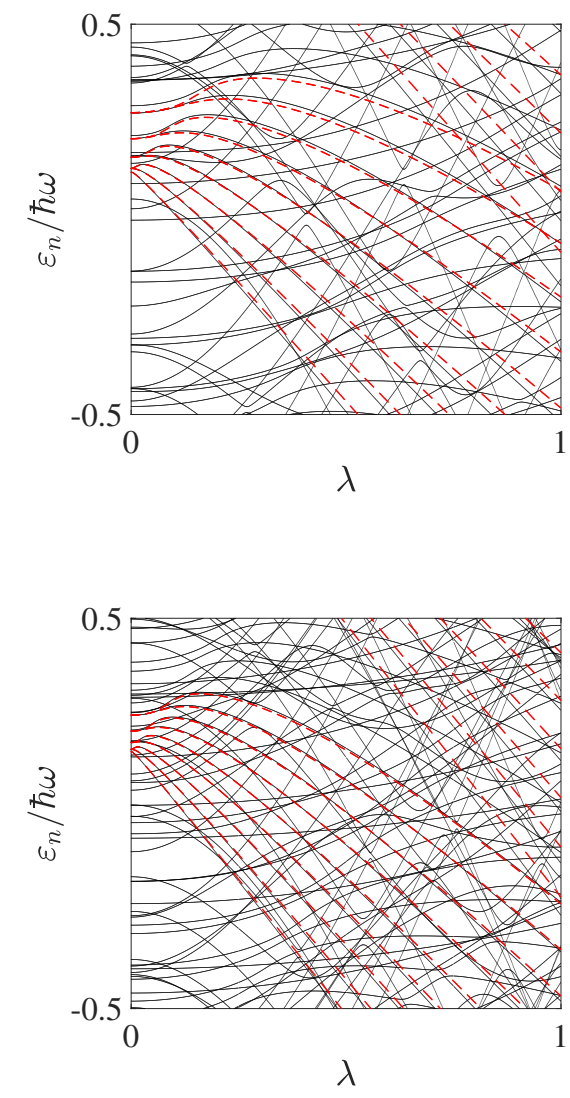

Figure 1: One Brillouin zone of quasienergies for the driven anharmonic oscilllator (2) with $\beta=0.1$ versus the scaled driving strength $\lambda$. Black lines represent numerically computed data; red dashed lines indicate the Mathieu approximation (27). The resonant states are $r=10$ ( $v=1.4508$; top) and $r=20(v=1.6909$; bottom).

state is strengthened further by resorting to a semiclassical viewpoint. As has been discussed by Breuer and Holthaus [30], a semiclassical approximation to the quasienergies and Floquet states of periodically driven, classically integrable $1+1$-dimensional systems can be obtained with the help of the Poincaré-Cartan form $\omega^{1}=p \mathrm{~d} x-H \mathrm{~d} t$ [31]: choosing a closed path $\gamma_{1}$ which winds once around an invariant $T$-periodic tube in the classical $(p, x, t)$ space, quantization is achieved by means of the condition

$$
\oint_{\gamma_{1}} \omega^{1}=2 \pi \hbar\left(k+\frac{\text { ind } \gamma_{1}}{4}\right), \quad k=0,1,2, \ldots
$$

where ind $\gamma_{1}$ denotes the Maslov index of $\gamma_{1}$; requiring $\gamma_{1}$ to lie in a plane $t=$ const., and thus in a $(p, x)$ plane of the usual phase space, reproduces the familiar Bohr-Sommerfeld quantization rule for the energy eigenstates of time-independent systems. The quasienergies then are computed with the help of a further $T$-periodic 


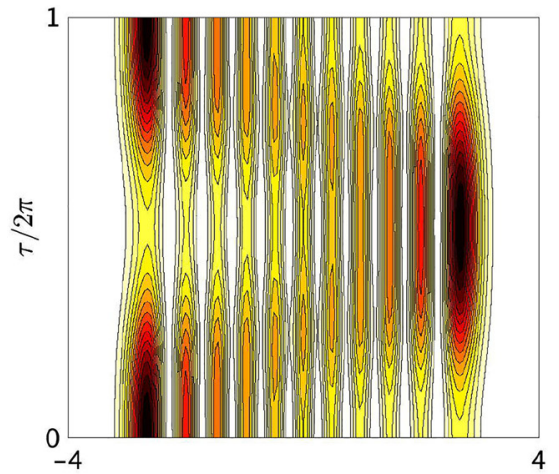

q

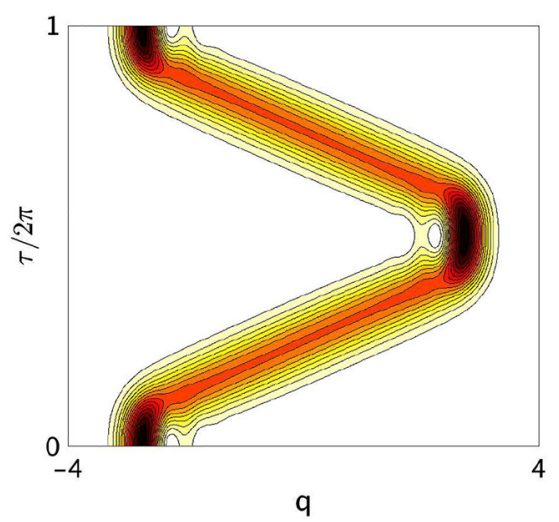

Figure 2: The contour plot of the probability density of the approximate Floquet state (26) with Mathieu quantum number $k=0$ of the driven anharmonic oscillator (2) with $\beta=0.9$ and $r=10$ ( $v=2.577$ ) for $\lambda=0.01$ (top) and $\lambda=1$ (bottom).

path which extends along such an invariant tube:

$$
\varepsilon=-\frac{1}{T} \int_{\gamma_{2}} \omega^{1}+\ell \hbar \omega, \quad \ell=0, \pm 1, \pm 2, \ldots
$$

Figure 3 shows phase-space portraits for the scaled Hamiltonian system (5) of the driven anharmonic oscillators, for both $v=1.05$ (top) and $v=1.4508$ (bottom). These figures depict sections of invariant tubes with the plane $\tau=0$, confirming that the classical dynamics are still mainly regular for the driving amplitudes considered here, so that the semiclassical rules (28) and (29) actually are applicable, identifying $\gamma_{1}$ with a matching closed curve in these figures. For $v=1.05$, these tubes surround the periodic orbit which originates from the stable fixed point of the undriven oscillator, so that the corresponding Floquet states represent perturbatively, $T$-periodically deformed energy eigenstates of the anharmonic oscillator, without change of their ordering. For $v=1.4508$ and $\lambda=0.9$,
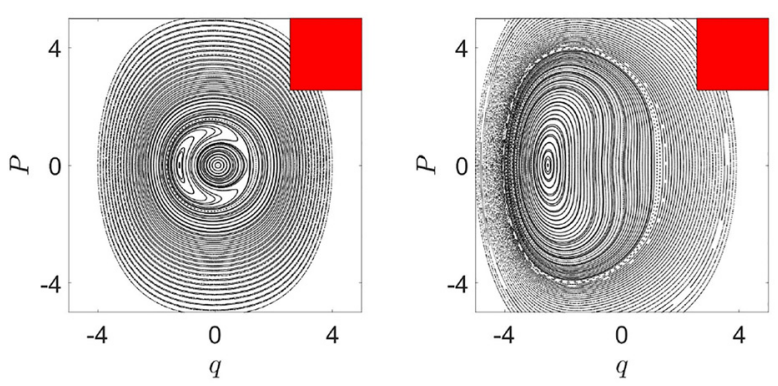

a) $\nu=1.05 ; \lambda=0.01$

b) $\nu=1.05 ; \lambda=0.9$
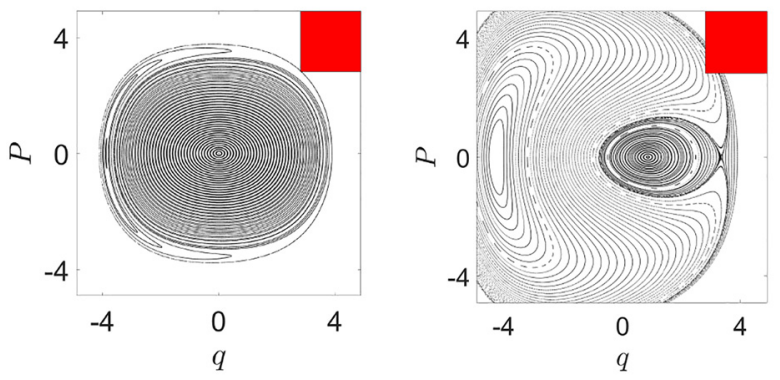

c) $\nu=1.4508 ; \lambda=0.01$

d) $\nu=1.4508 ; \lambda=0.9$

Figure 3: Phase-space portraits for a classical driven anharmonic oscillator (5) with $\beta=0.1$ for weak (left: $\lambda=0.01$ ) and strong (right: $\lambda=0.9$ ) driving. Panels (a) and (b) refer to nonresonant driving with $v=1.05$, whereas (c) and (d) visualize resonant driving with $v=1.4508$. The squares in the upper right corners indicate the area $2 \pi \hbar_{\text {eff }}=2 \pi \nu^{-1}$ semiclassically occupied by one Floquet state.

however, most of the invariant tubes surround the new stable $T$-periodic orbit which is created by the $1: 1$ resonance; these tubes do not result from the previous tubes by continuous deformation. Therefore, when applying the rule (28) to these new resonance-induced tubes, and selecting the matching ones for $k=0,1,2, \ldots$, a "new order" establishes itself, with the number of states which are accessible to the Mathieu approximation being given roughly by the number of quantized tubes which fit into the classical resonance zone.

\section{Quasi-stationary Floquet-state occupation probabilities}

Now the driven anharmonic oscillator is assumed to interact with an infinite phonon bath modeled by thermally occupied harmonic oscillators, the frequencies of which are distributed according to a spectral density $J(\widetilde{\omega})$. In order to describe the coupling of the anharmonic oscillator to this 
bath, a natural choice is

$$
H_{\mathrm{int}}=\gamma x \sum_{\widetilde{\omega}}\left(b_{\widetilde{\omega}}+b_{\widetilde{\omega}}^{\dagger}\right),
$$

where $b_{\widetilde{\omega}}^{\dagger}\left(b_{\widetilde{\omega}}\right)$ creates (annihilates) a phonon with frequency $\widetilde{\omega}$, accompanying a dipole-type transition among the Floquet states, and the coupling constant $\gamma$ carries the dimension of energy per length. Adopting the Born-Markov scheme worked out by Breuer et al. [7], the rate $\Gamma_{\mathrm{fi}}$ of bath-induced transitions from an initial anharmonic-oscillator Floquet state $i$ to a final one $f$ is written as a sum of partial rates,

$$
\Gamma_{\mathrm{fi}}=\sum_{\ell} \Gamma_{\mathrm{fi}}^{(\ell)}
$$

where these partial rates take the form

$$
\Gamma_{\mathrm{fi}}^{(\ell)}=\frac{2 \pi}{\hbar^{2}}\left|V_{\mathrm{fi}}^{(\ell)}\right|^{2} N\left(\omega_{\mathrm{fi}}^{(\ell)}\right) J\left(\left|\omega_{\mathrm{fi}}^{(\ell)}\right|\right) .
$$

Here, the index $\ell$ labels the different transition frequencies between the Floquet states under consideration,

$$
\omega_{\mathrm{fi}}^{(\ell)}=\left(\varepsilon_{\mathrm{f}}-\varepsilon_{\mathrm{i}}\right) / \hbar+\ell \omega \quad \text { with } \ell=0, \pm 1, \pm 2, \ldots,
$$

once again reflecting the Brillouin-zone structure of the quasienergy spectrum. The numbers $N(\widetilde{\omega})$ are given by the thermal occupation numbers of the phonon modes at the inverse bath temperature $\beta_{\text {bath }}=\left(k_{\mathrm{B}} T_{\text {bath }}\right)^{-1}$, with $k_{\mathrm{B}}$ denoting Boltzmann's constant, namely [7],

$$
N(\widetilde{\omega})=\left\{\begin{array}{lll}
\frac{1}{\exp \left(\beta_{\mathrm{bath}} \hbar \widetilde{\omega}\right)-1} & ; & \widetilde{\omega}>0 \\
\frac{1}{1-\exp \left(\beta_{\mathrm{bath}} \hbar \widetilde{\omega}\right)} & ; & \widetilde{\omega}<0
\end{array}\right.
$$

Finally, the quantities $V_{f i}^{(\ell)}$ appearing in the partial rates (32) are given by the Fourier components of the Floquet transition matrix elements,

$$
\left\langle u_{\mathrm{f}}(t)|\gamma x| u_{\mathrm{i}}(t)\right\rangle=\sum_{\ell} \mathrm{e}^{\mathrm{i} \ell \omega t} V_{\mathrm{fi}}^{(\ell)} .
$$

Having computed the total transition rates (31) in this manner, the quasi-stationary distributions $\left\{p_{n}\right\}$ of Floquetstate occupation probabilities are determined by solving the master equation

$$
0=\sum_{m}\left(\Gamma_{n m} p_{m}-\Gamma_{m n} p_{n}\right) .
$$

The numerical results described in the following two sections have been obtained by implementing this algortithm within a basis set of $b=61$ harmonic-oscillator eigenfunctions.

\section{Periodic Thermodynamics of Mathieu states}

For vanishing driving force, that is, for $\lambda=0$, the energy eigenstates of the anharmonic oscillator are occupied according to the canonical distribution

$$
P_{n}=\frac{\exp \left(-\beta_{\text {bath }} E_{n}\right)}{\sum_{m} \exp \left(-\beta_{\text {bath }} E_{m}\right)} .
$$

Therefore, when considering the Floquet-state occupation probabilities $p_{n}$ for $\lambda \neq 0$, I divide these probabilities by $P_{0}$, in order to get a good feeling for the magnitude of the observed effects. In particular, this scaling immediately indicates whether or not certain Floquet states carry more population than the ground state of the undriven anharmonic oscillator.

Figure 4 shows the Floquet-state occupation probabilities for anharmonicity $\beta=0.1$ and a bath with Ohmic
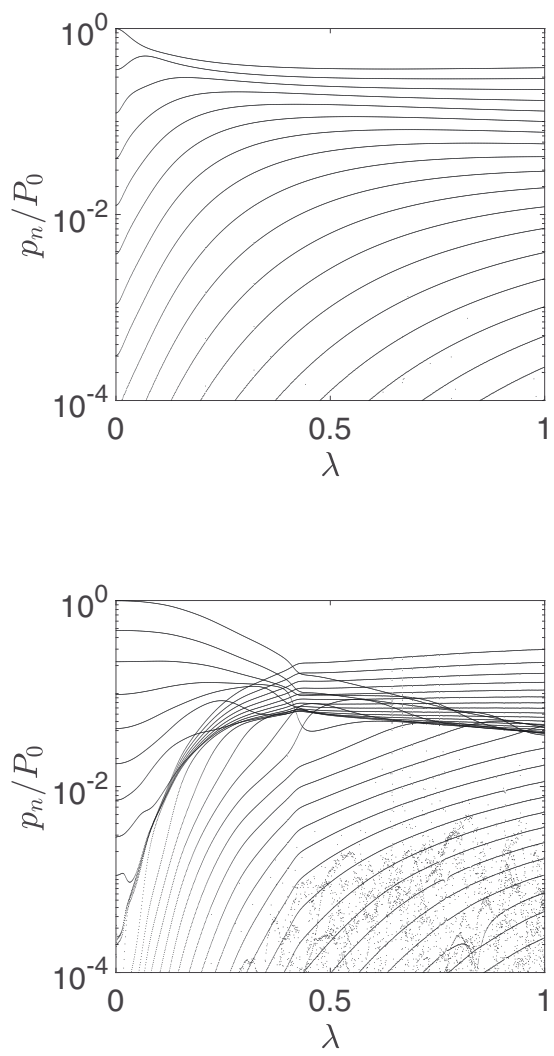

Figure 4: Floquet-state occupation probabilities $p_{n} / P_{0}$ for a driven anharmonic oscillator (2) with $\beta=0.1$ in a bath with scaled temperature $k_{\mathrm{B}} T_{\text {bath }} /(\hbar \omega)=1.0$ versus the driving strength $\lambda$ for nonresonant driving with $v=1.05$ (top), and resonant driving with $v=1.4508$ (bottom). An Ohmic bath density of states $J(\widetilde{\omega}) \propto \widetilde{\omega}$ is assumed here. 
density of states, $J(\widetilde{\omega}) \propto \widetilde{\omega}$, and scaled bath temperature $k_{\mathrm{B}} T_{\text {bath }} /(\hbar \omega)=1.0$ as functions of the driving strength $\lambda$ for two rather distinct cases: nonresonant driving with $v=1.05$ (top), and resonant driving with $v=1.4508$ (bottom). Under nonresonant driving the occupation probability of the Floquet state originating from the lowest energy eigenstate decreases with increasing $\lambda$, whereas the other occupation probabilities tend to increase, in accordance with an intuitive notion of "heating". Importantly, the graphs of the individual functions $p_{n}(\lambda)$ do not cross: the occupation probabilities retain their original order when the nonresonant drive is turned on, and made stronger.

Under resonant driving, however, an altogether different picture is observed. Now a certain group of Floquet states which carry only small populations for low driving amplitudes acquires relatively high populations in the strong-forcing regime. In parallel, the occupation probability of the Floquet state connected to the anharmonicoscillator ground states drops even below that of the members of this group. Most significantly, the Floquet states hosting most of the population for $\lambda \approx 1$ can be identified as those states which are described by the Mathieu approximation (26), such that the Mathieu quantum number $k$ determines their ordering: the Floquet state with $k=0$ which stems from the ground state of the pendulum (19) bears most of the population, the states with $k \geq 1$ successively less. Hence, a quantum resonance can systematically introduce a new order in a periodically driven open quantum system, expressed by the Mathieu quantum number $k$ which replaces the original quantum number $n$.

Keeping the parameters $\beta=0.1, \nu=1.4508$ and $\lambda=$ 0.9 fixed, Figure 5 depicts the Floquet-state occupation probabilities $p_{n} / P_{0}$ for varying inverse bath temperature $\beta_{\text {bath }} \hbar \omega=\hbar \omega /\left(k_{\mathrm{B}} T_{\text {bath }}\right)$, again assuming an Ohmic bath density of states. The most important observation here is that the occupation probabilities do not chance their order when the bath temperature changes. Therefore, even in the limit of very small bath temperatures the Mathieu ground state $k=0$ is expected to hold the highest population, as is characteristic of a true ground state. However, whereas the canonical ground-state occupation probability $P_{0}$ of the undriven oscillator inevitably approaches unity for $\hbar \omega /\left(k_{\mathrm{B}} T_{\text {bath }}\right) \rightarrow \infty$, several states of the Mathieu hierarchy appear to remain significantly populated in this limit.

\section{Mathieu quantum engineering}

Whereas the canonical distribution (37) is universal, that is, does not depend on the details of the system-bath coupling,

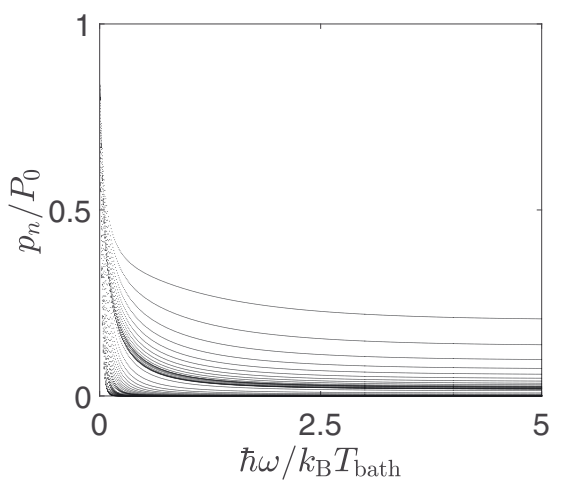

Figure 5: Floquet-state occupation probabilities $p_{n} / P_{0}$ for a driven anharmonic oscillator (2) with $\beta=0.1$ under resonant driving with $\nu=1.4508$ and $\lambda=0.9$, versus the scaled inverse bath temperature $\hbar \omega /\left(k_{\mathrm{B}} T_{\text {bath }}\right)$. The bath density of states is Ohmic.

this is different for the quasi-stationary distributions $\left\{p_{n}\right\}$ of Floquet-state occupation probabilities [7, 8]. This noteworthy fact allows one to "engineer" such distributions by willfully modifying the properties of the bath.

For the purpose of illustration, in this section I resort to spectral densities having the form of power functions with an exponent $c$,

$$
J(\widetilde{\omega}) \propto \widetilde{\omega}^{c},
$$

so that $c=1$ reproduces the Ohmic density, $c<1$ gives subOhmic densities, and $c>1$ describes super-Ohmic bath densities of states. In Figure 6, I plot the Floquet-state occupation probabilities computed for $\beta=0.1, v=1.4508$, $\lambda=0.9$, and $k_{\mathrm{B}} T_{\text {bath }} /(\hbar \omega)=1.0$ as functions of the exponent $c$. With the exception of an anomalous state which will not be discussed here, the ordering of the occupation

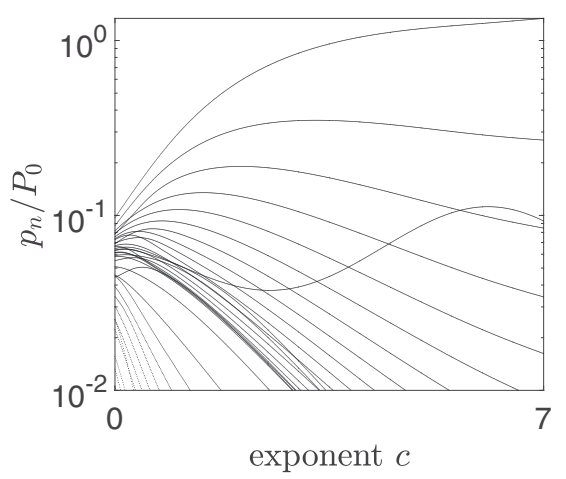

Figure 6: Floquet-state occupation probabilities $p_{n} / P_{0}$ for a resonantly driven anharmonic oscillator (2) with $\beta=0.1$, $v=1.4508$, and $\lambda=0.9$, versus the exponent $c$ of the spectral density $J(\widetilde{\omega}) \propto \widetilde{\omega}^{c}$ of the bath. 
probabilities is not significantly affected by the exponent; in particular, the Mathieu ground state retains its status as the Floquet state with the highest occupation probability for all values of $c$ considered. Most interestingly, $p_{k=0} / P_{0}$ increases substantially with $c$, such that $p_{k=0}>P_{0}$ for $c>4$ : in the presence of a bath with appropriate properties, the occupation of the effective Floquet ground state in the strong-driving regime can even exceed the occupation of the actual ground state of the undriven oscillator, with the bath having the same temperature in both cases.

This phenomenon, that is, the enhancement of the population of the resonance-induced effective Floquet ground state over that of the undriven true ground state, is illustrated further in Figure 7. There I use a superOhmic density with $c=6$, and study the dependence of the quasi-stationary distribution on the driving amplitude (top), and on the bath temperature (bottom). In response to an increasing driving amplitude one again observes the now familiar reordering previously found in Figure 4 for resonant driving, but here this Mathieu-state reordering is even more pronounced, resulting in $p_{k=0}>P_{0}$ for $\lambda \approx 1$.
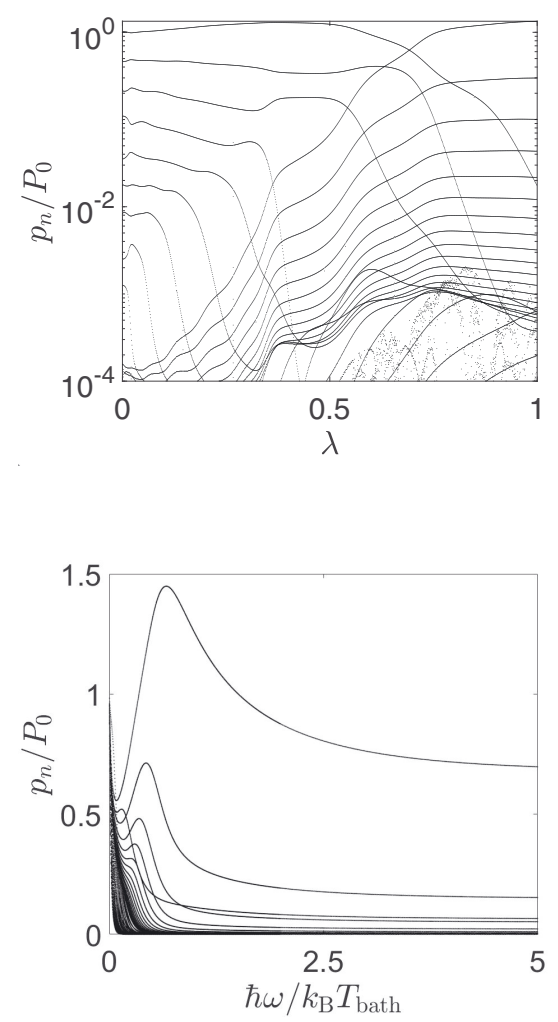

Figure 7: Floquet-state occupation probabilities $p_{n} / P_{0}$ for a resonantly driven anharmonic oscillator (2) with $\beta=0.1$, $\nu=1.4508$, and spectral bath density $J(\widetilde{\omega}) \propto \widetilde{\omega}^{6}$, versus the scaled driving strength $\lambda$ for $\hbar \omega /\left(k_{\mathrm{B}} T_{\text {bath }}\right)=1.0$ (top), and versus the scaled inverse bath temperature $\hbar \omega /\left(k_{\mathrm{B}} T_{\text {bath }}\right)$ for $\lambda=0.9$ (bottom).
Another remarkable observation is made when $\lambda=0.9$ is kept fixed, and the bath temperature is varied: the maximum enhancement of the population of the $k=0$ state occurs for an intermediate bath temperature, and is diminished when the bath is made colder.

\section{Conclusion}

The periodically driven anharmonic oscillator (1) provides a rich model system for studying the ramifications of quantum resonances in Periodic Thermodynamics. While the Mathieu approximation and the appearance of a state ordering according to the Mathieu quantum number $k$ have been worked out before $[18,19]$, here I have shown their implications for the quasi-stationary distributions of the Floquet-state occupation probabilities which establish themselves when the driven system is coupled to a heat bath [7]. In particular, I have demonstrated that the Mathieu state $k=0$ can actually play the role of an effective Floquet ground state, in the sense that it carries the highest population in the nonequilibrium steady state. Interestingly, this population does not necessarily go to unity when the bath temperature vanishes. Moreover, the populations of the near-resonant Floquet states which are described by the Mathieu approximation decrease successively with increasing Mathieu quantum number.

From the semiclassical viewpoint, is should be noted that the resonance-induced order requires that the classical dynamics inside the resonance zones in the classical phase space are still mainly regular, so that the corresponding Floquet states are associated with tubes which are invariant under the Hamiltonian flow, allowing the use of the quantization rules (28) and (29). This classical regularity is reflected by the fact that the approximate quasienergies (27) depend smoothly on the driving amplitude. However, outside the regular resonance zones the classical dynamics may be chaotic, confronting one with the classical-quantum correspondence for partly chaotic systems [32], leading to intricate quasienergy spectra featuring many large avoided crossings. Some traces of the resulting complications can be seen in the lower panel of Figure 4 when inspecting the occupation probabilities of those Floquet states which fall outside the domain of the Mathieu approximation; these occupation probabilties vary with the driving amplitude in a highly irregular manner.

Of particular interest is the observation that a proper choice of the parameters of the driving force, together with a suitable tayloring of the properties of the bath, systematically leads to occupation probabilities of the effective 
Floquet ground state $k=0$ which are appreciably higher than the thermal occupation probability of the system's true ground state in the absence of the driving force. It is an open and challenging question whether a similar effect could also occur in periodically driven many-body systems which interact with a heat bath.

Acknowledgments: This work has been supported by the Deutsche Forschungsgemeinschaft (DFG, German Research Foundation) through Project No. 397122187. The numerical computations were performed on the highperformance cluster CARL of the University of Oldenburg, funded by the DFG and the Ministry of Science and Culture (MWK) of the State of Lower Saxony.

Author contribution: The author has accepted responsibility for the entire content of this submitted manuscript and approved submission.

Research funding: Funded by the Deutsche Forschungsgemeinschaft (DFG, German Research Foundation) through Project No. 397122187.

Conflict of interest statement: The author declares no conflicts of interest regarding this article.

\section{References}

[1] D. V. Else, B. Bauer, and C. Nayak, "Floquet time crystals," Phys. Rev. Lett., vol. 117, 2016, Art no. 090402.

[2] N. Y. Yao, A. C. Potter, I.-D. Potirniche, and A. Vishwanath, "Discrete time crystals: rigidity, criticality, and realizations," Phys. Rev. Lett., vol. 118, 2017, Art no. 030401.

[3] J. Zhang, P. W. Hess, A. Kyprianidis, et al., "Observation of a discrete time crystal," Nature, vol. 543, p. 217, 2017.

[4] S. Choi, J. Choi, R. Landig, et al., "Observation of discrete time-crystalline order in a disordered dipolar many-body system," Nature, vol. 543, p. 221, 2017.

[5] R. Graham and R. Hübner, "Generalized quasi-energies and Floquet states for a dissipative system," Ann. Phys., vol. 234, p. 300, 1994.

[6] S. Kohler, Th. Dittrich, and P. Hänggi, "Floquet-Markovian description of a parametrically driven, dissipative harmonic quantum oscillator," Phys Rev. E, vol. 55, p. 300, 1997.

[7] H.-P. Breuer, W. Huber, and F. Petruccione, "Quasistationary distributions of dissipative nonlinear quantum oscillators in strong periodic driving fields," Phys. Rev. E, vol. 61, p. 4883, 2000.

[8] W. Kohn, "Periodic thermodynamics," J. Stat. Phys., vol. 103, p. 417, 2001.

[9] D. W. Hone, R. Ketzmerick, and W. Kohn, "Statistical mechanics of Floquet systems: the pervasive problem of near degeneracies," Phys. Rev. E., vol. 79, 2009, Art no. 051129.

[10] R. Ketzmerick and W. Wustmann, "Statistical mechanics of Floquet systems with regular and chaotic states," Phys. Rev. E, vol. 82, 2010, Art no. 021114.

[11] M. Langemeyer and M. Holthaus, "Energy flow in periodic thermodynamics,” Phys. Rev. E, vol. 89, 2014, Art no. 012101.
[12] T. Shirai, T. Mori, and S. Miyashita, "Condition for emergence of the Floquet-Gibbs state in periodically driven open systems," Phys. Rev. E, vol. 91, 2015, Art no. 030101(R).

[13] D. E. Liu, "Classification of the Floquet statistical distribution for time-periodic open systems," Phys. Rev. B, vol. 91, p. $144301,2015$.

[14] H.-J. Schmidt, J. Schnack, and M. Holthaus, "Periodic thermodynamics of the Rabi model with circular polarization for arbitrary spin quantum numbers," Phys. Rev. E, vol. 100, 2019, Art no. 042141.

[15] O. R. Diermann, H. Frerichs, and M. Holthaus, “Periodic thermodynamics of the parametrically driven harmonic oscillator," Phys. Rev. E, vol. 100, 2019, Art no. 012102.

[16] O. R. Diermann and M. Holthaus, "Floquet-state cooling," Sci. Rep., vol. 9, p. 17614, 2019.

[17] O. R. Diermann, H.-J. Schmidt, J. Schnack, and M. Holthaus, “Environment-controlled Floquet-state paramagnetism," Phys. Rev. Res., vol. 2, 2020, Art no. 023293.

[18] J. Henkel and M. Holthaus, "Classical resonances in quantum mechanics," Phys. Rev. A, vol. 45, p. 1978, 1992.

[19] M. Holthaus, "On the classical-quantum correspondence for periodically time dependent systems," Chaos, Solit. Fractals, vol. 5, p. 1143, 1995.

[20] Ya. B. Zel'dovich, "The quasienergy of a quantum-mechanical system subjected to a periodic action," J. Exp. Theor.

Phys., vol. 51, p. 1492,

1966, [Sov. Phys. JETP 24, 1006 (1967)].

[21] V. I. Ritus, "Shift and splitting of atomic energy levels by the field of an electromagnetic wave," J. Exp. Theor. Phys., vol. 51, p. 1544, 1966, [Sov. Phys. JETP 24, 1041 (1967)].

[22] H. Sambe, "Steady states and quasienergies of a quantum-mechanical system in an oscillating field," Phys. Rev. A, vol. 7, p. 2203, 1973.

[23] M. Combescure, "The quantum stability problem for some class of time-dependent Hamiltonians," Ann. Phys., vol. 185, p. 86, 1988.

[24] J. S. Howland, “Quantum stability," in Schrödinger Operators: The Quantum Mechanical Many-Body Problem, Lecture Notes in Physics, vol. 403, Berlin, Springer, 1992, p. 100.

[25] J. S. Howland, "Stability of quantum oscillators," J. Phys. Math. Gen., vol. 25, p. 5177, 1992.

[26] B. V. Chirikov, "A universal instability of many-dimensional oscillator systems," Phys. Rep., vol. 52, p. 263, 1979.

[27] G. P. Berman and G. M. Zaslavsky, "Theory of quantum nonlinear resonance," Phys. Lett., vol. 61, p. 295, 1977.

[28] B. Gertjerenken and M. Holthaus, "Trojan quasiparticles," New J. Phys., vol. 16, 2014, Art no. 093009.

[29] M. Abramowitz and I. A. Stegun, Eds., Handbook of Mathematical Functions New York, Dover Publications, 1970. chap. 20.

[30] H. P. Breuer and M. Holthaus, "A semiclassical theory of quasienergies and Floquet wave functions," Ann. Phys., vol. 211, p. 249, 1991.

[31] V. I. Arnold, Mathematical Methods of Classical Mechanics, in Graduate Texts in Mathematics, vol. 60, New York, Springer-Verlag, 1989.

[32] M. C. Gutzwiller, Chaos in Classical and Quantum Mechanics, in Interdisciplinary Applied Mathematics, vol. 1, New York, Springer-Verlag, 1990. 